\title{
A cohesive model of fatigue of ferroelectric materials under electro- mechanical cyclic loading
}

Irene Arias, Santiago Serebrinsky, Michael Ortiz

Irene Arias, Santiago Serebrinsky, Michael Ortiz, "A cohesive model of fatigue of ferroelectric materials under electro-mechanical cyclic loading," Proc. SPIE 5387, Smart Structures and Materials 2004: Active Materials: Behavior and Mechanics, (21 July 2004); doi: 10.1117/12.540097

SPIE. Event: Smart Structures and Materials, 2004, San Diego, CA, United States 


\title{
A cohesive model of fatigue of ferroelectric materials under electro-mechanical cyclic loading
}

\author{
I. Arias, S. Serebrinsky and M. Ortiz \\ Division of Engineering and Applied Science \\ California Institute of Technology \\ 1200 E. California Blvd., Pasadena, USA
}

\begin{abstract}
A cohesive fatigue-crack nucleation and growth model for ferroelectric materials under electro-mechanical loading is presented. The central feature of the model is a hysteretic cohesive law which couples the mechanical and electrical fields. This law can be used in conjunction with general constitutive relations of bulk behavior, possibly including domain switching, in order to predict fatigue crack growth under arbitrary loading conditions. Another appealing feature of the model is its ability to predict fatigue-crack nucleation. Despite the scarcity and uncertainty of the experimental data, comparisons with PZT fatigue-life data are encouraging.
\end{abstract}

Keywords: Fatigue, nucleation, ferroelectrics, electric loading, mechanical loading

\section{INTRODUCTION}

Ferroelectric materials are extensively used in a variety of sensor and actuator applications, as well as in nonvolatile ferroelectric memory devices. However, ferroelectrics are brittle, and their low fracture toughness (in the order of $1 \mathrm{MPa} \mathrm{m}{ }^{1 / 2}$ ) makes them susceptible to cracking. In particular, under cyclic electrical loading, ferroelectric materials exhibit electrical fatigue (loss of switchable polarization) and, due to the strong electromechanical coupling, mechanical fatigue as well. Thus, the propagation of fatigue cracks hinders the performance of the devices and raises serious reliability concerns.

Ferroelectric fatigue is caused by a combination of electrical, mechanical and electrochemical processes, each of which has been claimed to be responsible for fatigue. ${ }^{1}$ Electrochemical mechanisms include pinning of domain walls by injected charges from the electrodes or oxygen vacancies. The role of the latter in ferroelectric fatigue has been extensively studied. ${ }^{2}$ Purely mechanical mechanisms, such as microcracking, are also likely to play some role. Thus, fatigued ceramic specimens often contain scattered microcracks of size comparable to that of the grains. ${ }^{3,4}$ Profuse microcrack clouds have been observed at the specimen edges and surrounding macroscopic cracks. ${ }^{5}$ Macroscopic crack patterns are present in some fatigued ceramic specimens. ${ }^{6}$ Severe cracking was also observed in barium titanate single crystals subjected to cyclic bipolar electric load ${ }^{7}$ (cf Fig. 1). Modelling work suggests that microcracking is indeed a cause of loss of polarization. ${ }^{8}$ Despite these advances, the precise chain of events leading to fatigue in ferroelectrics has not been elucidated to date.

Experiments on crack propagation have been reported for samples loaded electrically, mechanically, or under combined loading, cyclic or static. ${ }^{9-12}$ It is not uncommon for different experiments to lead to apparently contradictory conclusions, ${ }^{13}$ a testament to the complexity of the phenomenon of ferroelectric fatigue. The fracture toughness has been shown to be anisotropic with respect to the poling direction. ${ }^{12}$ The apparent toughness observed in ferroelectrics has also been shown to strongly depend on the direction of the applied electric field with respect to poling and crack orientation. ${ }^{13,14}$ Localized domain switching at the crack tip has been suggested as a basic mechanism that controls the fracture toughness of ferroelectrics. Thus, the concentrated stress or electric field at the crack tip induces local switching that can either shield or promote crack growth depending on the loading conditions. ${ }^{15-18}$ Other conditions or features that have been shown to have a strong effect on the crack propagation are the electrical conditions of the crack faces, the grain size, and porosity, to name a salient few.

Further author information: Send correspondence to Michael Ortiz: E-mail: ortiz@aero.caltech.edu, Telephone: 1 (626) 395-4530. 

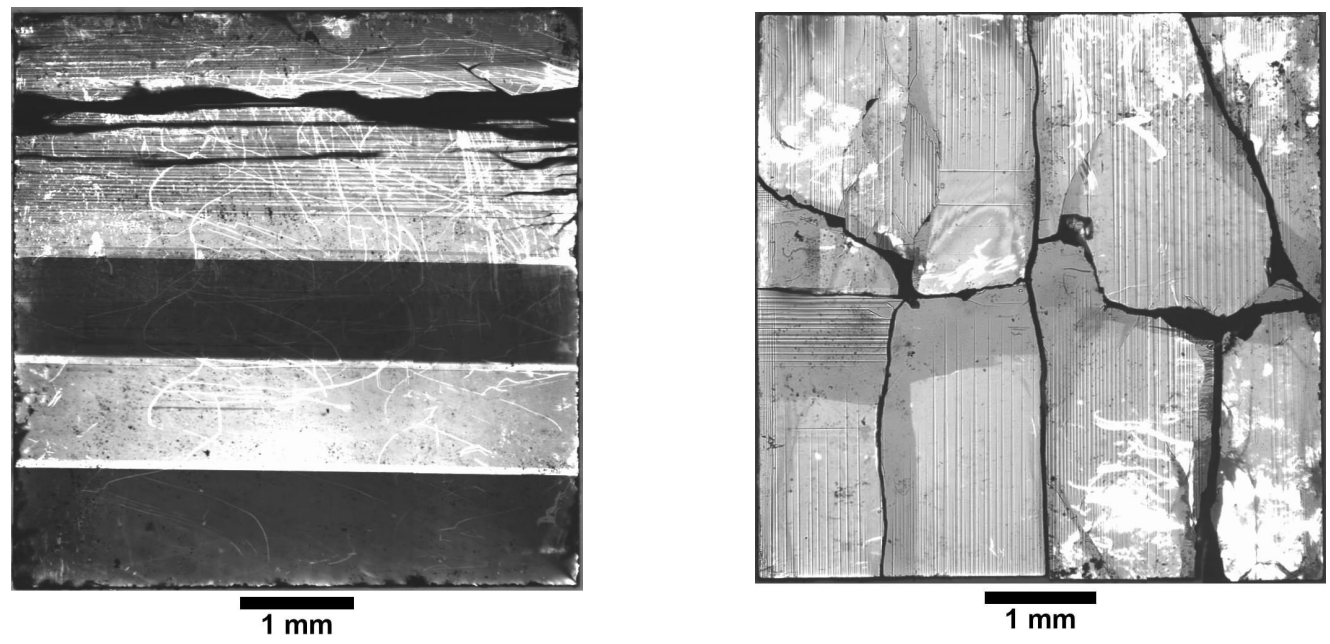

Figure 1. Pictures of damaged barium titanate crystals after repeated electrical cycling. ${ }^{7}$

Some early models of fracture of ferroelectrics were based on linear piezoelectric fracture mechanics. ${ }^{19}$ However, this approach results in predictions contrary to observation, e. g., as regards the effect of the applied electric field on crack propagation, or the fatigue crack growth under cyclic electrical loading. ${ }^{20}$ It is now accepted that the nonlinearity in the response of ferroelectrics plays an essential role in determining their fracture properties. $^{13,21,22}$ In an effort to account for such nonlinearity, Zhu and Yang ${ }^{15-17}$ developed a theory of fracture based on the concept of small scale switching. In this theory, the constitutive relation is assumed to be linear and, following Hwang and coworkers, ${ }^{23}$ and nonlinearity is introduced by means of an ad hoc switching criterion. The effective behavior of the small-scale switched regions is then estimated by means of a Reuss-type approximation. This theory has successfully overcome some of the shortcomings of linear piezoelectric fracture mechanics. An alternative approach is provided by the so-called strip saturation model of Gao and coworkers. ${ }^{24-26}$ This phenomenological model assumes that the crack is mechanically brittle but electrically ductile, and adopts a Dugdale-like model for the electric displacement. Although this model explains a number experimental observations, its physical basis has been called into question. ${ }^{22}$ The effect of the electrical boundary conditions on the crack faces is also a subject of controversy, and it has been suggested that it may strongly influence the fracture behavior. ${ }^{20,22}$

Cohesive theories provide an effective means of modelling fatigue-crack nucleation and growth for arbitrary crack and specimen geometries and loading histories. ${ }^{27,28}$ In particular, cohesive models of fatigue account for deviations from Paris' law such as are observed for short cracks and overloads. ${ }^{27}$ Cohesive models of fatigue are also capable of predicting fatigue-crack nucleation in smooth-surfaced components. ${ }^{27}$ The appealing feature of cohesive models of fatigue is that a single mechanistic, albeit empirical, model applies to nucleation and propagation, short and long cracks, and arbitrary loading histories. In this note we present an extension of the fatigue model of Nguyen et al. ${ }^{27}$ to fatigue of ferroelectrics under electro-mechanical loading. The present note is extracted and condensed from Ref. 29.

The organization of the note is as follows. In Section 3 the proposed cohesive fatigue law is presented, and some properties of this law under cyclic loading are derived. In Section 4 the model is applied to crack nucleation and the calculated fatigue diagrams are compared with experiment. We sum up and close in Section 5. 


\section{THE MECHANICS OF DECOHESION IN FERROELECTRICS}

The essential structure of cohesive laws in ferroelectrics may be elucidated by recourse to a conventional ColemanNoll argument (cf Ref. 30 for an application to mechanical decohesion). In particular, the pairing between stress-like and deformation-like variables is determined by the internal-power identity or, equivalently, by the virtual-work identity. For a dielectric solid, this identity is ${ }^{19,31,32}$

$$
\int_{\Omega}(\boldsymbol{\sigma}: \delta \boldsymbol{\varepsilon}-\mathbf{D} \cdot \delta \mathbf{E}) d V=\int_{\Omega}(\boldsymbol{b} \cdot \delta \boldsymbol{u}-\rho \delta \phi) d V+\int_{\partial \Omega}(\boldsymbol{t} \cdot \delta \boldsymbol{u}-\omega \delta \phi) d S,
$$

where $\Omega$ is the spatial domain occupied by the solid and $\partial \Omega$ is its boundary; $\boldsymbol{\sigma}$ is the stress; $\boldsymbol{\varepsilon}$ is the strain; $\mathbf{D}$ is the electric displacement; $\mathbf{E}=-\nabla \phi$ is the electric field; $\boldsymbol{b}$ is the body force; $\boldsymbol{u}$ is the displacement; $\rho$ is the free charge density; $\phi$ is the electric potential; $\boldsymbol{t}$ is the surface traction; and $\omega=-\mathbf{D} \cdot \boldsymbol{n}$ is the surface charge per unit area. For a solid with a surface of discontinuity $\Gamma$, or cohesive surface, we have

$$
\begin{aligned}
\delta W_{\mathrm{ext}} & =\int_{\Omega}(\boldsymbol{b} \cdot \delta \boldsymbol{u}-\rho \delta \phi) d V+\int_{\partial \Omega}(\boldsymbol{t} \cdot \delta \boldsymbol{u}-\omega \delta \phi) d S \\
& =\int_{\Omega}(\boldsymbol{b} \cdot \delta \boldsymbol{u}-\rho \delta \phi) d V+\int_{\partial \Omega}[(\boldsymbol{n} \cdot \boldsymbol{\sigma}) \cdot \delta \boldsymbol{u}+(\boldsymbol{n} \cdot \mathbf{D}) \delta \phi] d S \\
& =\int_{\Omega}(\boldsymbol{b} \cdot \delta \boldsymbol{u}-\rho \delta \phi) d V+\int_{\Omega^{ \pm}}[\nabla \cdot(\boldsymbol{\sigma} \cdot \boldsymbol{\delta} \boldsymbol{u})+\nabla \cdot(\mathbf{D} \delta \phi)] d V+\int_{\Gamma}[(\boldsymbol{n} \cdot \boldsymbol{\sigma}) \cdot \llbracket \delta \boldsymbol{u} \rrbracket+(\boldsymbol{n} \cdot \mathbf{D}) \llbracket \delta \phi \rrbracket] d S
\end{aligned}
$$

and consequently

$$
\delta W_{\mathrm{int}}=\int_{\Omega^{ \pm}}(\boldsymbol{\sigma}: \delta \boldsymbol{\varepsilon}-\mathbf{D} \cdot \delta \mathbf{E}) d V+\int_{\Gamma}[(\boldsymbol{n} \cdot \boldsymbol{\sigma}) \cdot \delta \llbracket \boldsymbol{u} \rrbracket+(\boldsymbol{n} \cdot \mathbf{D}) \delta \llbracket \phi \rrbracket] d S
$$

For monotonic loading of the cohesive surface, these work-conjugacy relations naturally suggest a free energy per unit surface, or cohesive potential, or the form $\Phi(\llbracket \boldsymbol{u} \rrbracket, \llbracket \phi \rrbracket, \boldsymbol{q})$ such that

$$
\begin{aligned}
\boldsymbol{t} & =\frac{\partial \Phi}{\partial \llbracket \boldsymbol{u} \rrbracket} \\
-\omega & =\frac{\partial \Phi}{\partial \llbracket \phi \rrbracket}
\end{aligned}
$$

where $\boldsymbol{q}$ is some suitable set of internal variables. A possible additional dependence of $\Phi$ on temperature is omitted for notational convenience. Thus, $\Phi$ depends both on the displacement and electric potential jumps across $\Gamma$ and acts as a potential jointly for the mechanical tractions and the surface charge density.

As already mentioned, some of the preceding ideas are partially present in the strip saturation model of Gao, ${ }^{24-26}$ which adopts a Dugdale-like model for the electric displacement. The present formulation allows for arbitrary decohesion laws and, in particular, for the coupling of the mechanical and electrical fields. It should also be carefully noted that the cohesive potential $\Phi(\llbracket \boldsymbol{u} \rrbracket, \llbracket \phi \rrbracket)$ represents the physics of decohesion of a surface of discontinuity, and it does not presume a particular form of the constitutive laws governing the behavior in the bulk. In particular, it is possible to apply the cohesive model in conjunction with Landau-Ginzburg-Devonshire models of domain switching (cf, e. g., Ref. 33). As already noted, the detailed boundary conditions on the crack faces are thought to greatly affect the fracture behavior of electrically driven crack growth. ${ }^{20}$ In particular, partial discharge or charge separation effects have been suggested to play an important role in the vicinity of the crack tip by McMeeking. ${ }^{22}$ The cohesive law provides a useful framework for accounting for these phenomena.

\section{COHESIVE LAWS FOR CYCLING LOADING}

As noted by Nguyen et al., ${ }^{27}$ reversible cohesive laws such as (3a) and (3b) do not predict crack advance under cyclic loading and, therefore, are insufficient for modelling fatigue. Instead, for a cohesive law to predict fatigue it must be irreversible and account for loading-unloading hysteresis. Loading-unloading irreversibility may be 


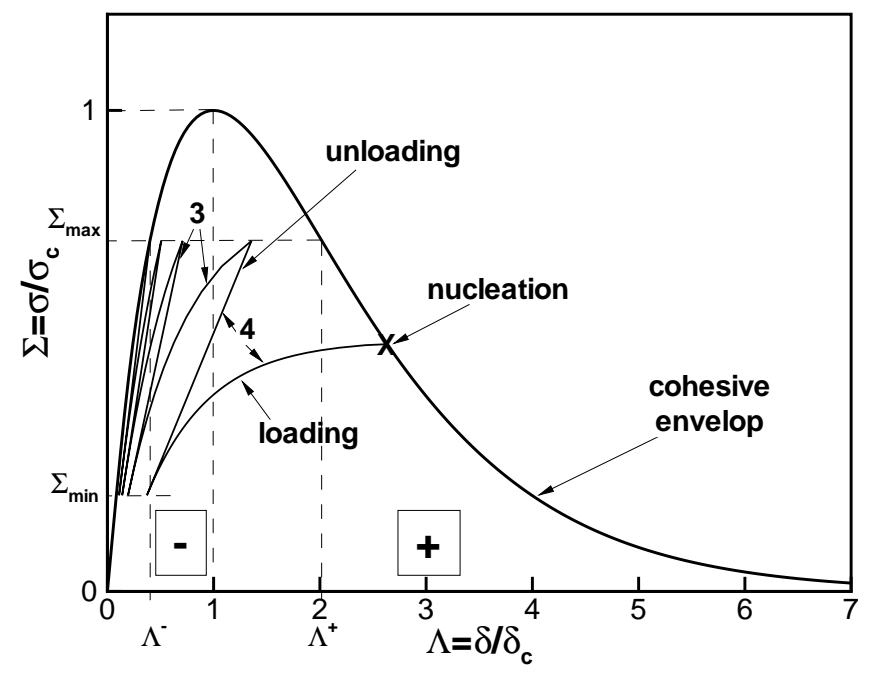

Figure 2. Cyclic behavior predicted by the model and conventional definition of fatigue initiation. $\sigma_{c}$ and $\delta_{c}$ are the critical traction and opening displacement, respectively. Numbers refer to the number of cycle.

built into a cohesive law by means of the internal variable formalism (cf, e. g., Ref. 30). The loading-unloading hysteresis model presented here follows that proposed by Nguyen et al. ${ }^{27}$ for mechanical fatigue.

Following Ref. 30, we begin by introducing an effective opening displacement of the form

$$
\delta=\sqrt{\delta_{n}^{2}+\beta^{2} \delta_{t}^{2}+\gamma^{2} \psi^{2}}
$$

where we write

$$
\begin{aligned}
& \delta_{n}=\llbracket \boldsymbol{u} \rrbracket \cdot \boldsymbol{n} \\
& \delta_{t}=\sqrt{|\llbracket \boldsymbol{u} \rrbracket|^{2}-\delta_{n}^{2}} \\
& \psi=\llbracket \phi \rrbracket
\end{aligned}
$$

for the normal and tangential components of the opening displacement and the electric potential jump, respectively. The parameters $\beta$ and $\gamma$ assign different relative weights to normal and tangential opening displacements, thus differentiating between mode I and modes II and III of fracture; and to opening displacements and the electric potential jump, thus differentiating between mechanical and electrical fatigue. The modelling process is greatly simplified by the assumption that the cohesive potential $\Phi$ depends on the displacement and electric potential jumps through the effective opening displacement $\delta$ only, i. e., by assuming

$$
\Phi=\Phi(\delta, \boldsymbol{q})
$$

An effective traction may also be defined as

$$
\sigma=\frac{\partial \Phi}{\partial \delta}
$$

Using the chain rule, (3a) and (3b) evaluate to

$$
\begin{aligned}
\boldsymbol{t} & =\frac{\sigma}{\delta}\left[\left(1-\beta^{2}\right) \delta_{n} \boldsymbol{n}+\beta^{2} \boldsymbol{\delta}\right] \\
-\omega & =\frac{\sigma}{\delta} \gamma^{2} \psi
\end{aligned}
$$

Next we further specialize the preceding framework along the lines of the cohesive model of fatigue crack nucleation and growth proposed by Serebrinsky and $\mathrm{Ortiz}^{28}$ and by Nguyen et al.. ${ }^{27}$ The essential feature to 
include in the model is loading-unloading hysteresis. The specific scheme chosen to build hysteresis into the model is illustrated in Fig. 2. Monotonic loading is characterized by a cohesive law $\sigma(\delta)$, referred to as the monotonic envelop, with or without an initial threshold. Unloading takes place from the unloading point $\left(\delta_{u}, \sigma_{u}\right)$ towards the origin of the $(\delta, \sigma)$ plane. The unloading slope is, therefore, $K_{u}=\sigma_{u} / \delta_{u}$, and is kept constant during each unloading step for simplicity. Upon reloading, we assume that the reloading slope $K_{l}=d \sigma / d \delta$ decreases with increasing opening displacement as a result of interfacial degradation mechanisms occurring at the microscale. For definiteness we assume

$$
\dot{K}_{l}=-K_{l} \frac{\dot{\delta}}{L}
$$

during loading, i. e., while $\dot{\delta}>0$. In the second of these equations $L$ is an intrinsic length of the material. Thus, the internal variables of the model are $\delta_{u}, \sigma_{u}$ and $K_{l}$, namely, the unloading opening displacement and traction and the reloading slope, respectively. The type of cyclic behavior predicted by the model is illustrated in Fig. 2.

For cycling between constant maximum and minimum tractions, $\sigma_{\max }$ and $\sigma_{\min }$, a straightforward calculation gives

$$
\delta_{n+1}=R \delta_{n}-L \log \left[1-(1-R) \delta_{n} / L\right]
$$

where in this relation $n$ is cycle number, $\delta_{n}$ denotes the value of $\delta_{u}$ after cycle $n$, and $R \equiv \sigma_{\min } / \sigma_{\max }$. Eq. 10 can now be applied recursively to determine the evolution of $\delta_{n}$. It is interesting to note that, the evolution law does not depend on $\sigma_{\max }$ and $\sigma_{\min }$ individually, but only on the ratio $R$.

\section{APPLICATION TO FATIGUE NUCLEATION}

An appealing feature of cohesive models of fatigue, such as just described, is that they are capable of describing fatigue-crack initiation. In this framework, initiation is understood to take place when the trajectory of the system in the $(\delta, \sigma)$ plane reaches the monotonic envelop $\sigma(\delta)$, Fig. 2. Suppose that this envelop is as shown in the figure, i. e., for every value of $\sigma$ there are exactly two values of $\delta$, which we denote $\delta^{-}(\sigma) \leq \delta^{+}(\sigma)$. An example of such law is the Universal Binding Energy Relation (UBER) of Rose et al. ${ }^{34}$ Note that, in a continuum framework, binding relations must be corrected to have an infinite slope at the origin in order to avoid double counting of the elasticity of the material. The procedure for effecting this correction has been described in detail by Rice. ${ }^{35}$ An asymptotic analysis ${ }^{28}$ of eq. 10 and its iterates then gives the number of cycles $N$ to nucleation as

$$
N \sim \frac{2 L}{(1-R)^{2}}\left(\frac{1}{\delta^{-}\left(\sigma_{\max }\right)}-\frac{1}{\delta^{+}\left(\sigma_{\max }\right)}\right)=N\left(\sigma_{\max }, R, \delta_{c} / L\right)
$$

The model thus predicts the number of cycles to nucleation to be a function of three parameters: the maximum effective traction $\sigma_{\max }$; the ratio $R \equiv \sigma_{\min } / \sigma_{\max }$; and the ratio of the critical effective opening displacement $\delta_{c}$ at which the maximum cohesive strength of the material is attained and the characteristic length $L$. We note from (11) that increasing $R$ shifts the $\sigma_{\max }-N$ curves to higher $N$, whereas decreasing $\delta_{c}$ has the same effect. It is interesting to note that relation (11) can predict threshold behavior. Thus, suppose that the monotonic envelop $\sigma(\delta)$ exhibits a threshold traction $\sigma_{0}$, i. e., $\delta^{-}=0$ for $\sigma \leq \sigma_{0}$. Then, it follows from (11) that $N=\infty$ for $\sigma_{\max } \leq \sigma_{0}$, i. e., nucleation requires that $\sigma_{\max }$ exceed the threshold value $\sigma_{0}$.

Common total life fatigue plots are Wöhler plots, namely, stress amplitude $S=\sigma_{\max }-\sigma_{\min } v s$. cycles to failure $N_{\mathrm{f}}$, for constant mean stress $\bar{\sigma}=\left(\sigma_{\max }+\sigma_{\min }\right) / 2$, also known as S-N plots. Two variants of these plots are often found in the literature, namely $S$ vs. $N_{\mathrm{f}}$ (or possibly $\log N_{\mathrm{f}}$ ) at constant $R$, and $\sigma_{\max } v s$. $N_{\mathrm{f}}$ at constant $R$ (e. g., Ref. 37). A comparison between the predictions of the model and experimental data for PZT is shown in Fig. 3. More detailed and extensive comparisons may be found in Ref. 29. Unfortunately, a thorough validation of the model is hampered by the scarcity and low quality of the data. In order to make contact with experiment, we assume that the number of cycles $N$ to initiation is close to the entire fatigue life $N_{\mathrm{f}}$ of the specimen. This assumption is plausible for smooth-surface specimens in the absence of pre-cracks. The adjustable parameters of the model are: the cohesive strength $\sigma_{c}$, the critical opening displacement $\delta_{c}$, the characteristic fatigue length $L$, the threshold traction $\sigma_{0}$ and $\gamma$. Figure 3a corresponds to zero applied mechanical stress and cyclic bipolar applied electric load $(\omega)$. Since in the experiments ${ }^{6}$ the controlled variable is the cyclic applied voltage between the electrodes $\Delta \phi$, which varies between $\pm \Delta \phi_{\max }$, we have run additional simulations to obtain the value of 



Figure 3. Computed number of cycles to fatigue-crack nucleation vs experiment for PZT. a) Commercial PZT PIC151, ${ }^{6}$ $\sigma_{0} / \sigma_{c}=0.54, \delta_{c} / L=0.08$, electrical loading. b) Commercial PZT, ${ }^{36} \sigma_{0} / \sigma_{c}=0.18, \delta_{c} / L=0.008$, mechanical loading ( $\tau_{\max }$ is the maximum applied traction).

$\omega_{\max }$ from $\pm \Delta \phi_{\max }$. These followed the scheme proposed in Ref. 33 . A value of $\gamma \sigma_{c}=0.52 \mathrm{C} / \mathrm{m}^{2}$ was used. Figure $3 \mathrm{~b}$ corresponds to cyclic mechanical loading at zero applied electric field, ${ }^{36}$ and $\sigma_{c}=250 \mathrm{MPa}$, which is of the order of the tensile strength of PZT, was used.

In view of the scatter and uncertainties in the data, the agreement between theory and observation is encouraging.

\section{CONCLUDING REMARKS}

We have presented an extension of the fatigue model of Nguyen et al. ${ }^{27}$ to fatigue of ferroelectrics under electromechanical loading. The central feature of the model is a hysteretic cohesive law which couples the mechanical and electrical fields. This law can be used, e. g., in finite-element calculations, in conjunction with general constitutive relations of bulk behavior, possibly including domain switching, in order to predict fatigue crack growth under arbitrary loading conditions. In metals, cohesive theories of fatigue-crack growth have demonstrated a remarkable ability to effect predictions beyond Paris-like behavior and capture the effect of small-crack sizes, overloads, and other 'anomalous' behaviors. ${ }^{27}$ Another appealing feature of cohesive theories of fracture is that they can predict fatigue-crack nucleation. Again, in the context of metals such predictions capture the salient experimental trends. $^{28}$

The present extension of this approach couples the mechanical and electrical fields by the simple device of introducing an effective opening displacement which combines, with different weights, the normal and tangential opening displacements and the electric potential jump. Despite the scarcity and uncertainty of the experimental data, the comparisons with experiment presented in this note, and other more extensive comparisons presented in Ref. 29, are encouraging. Of course, the model presented in this note is phenomenological in nature and many of its details owe to simple convenience. In addition, the parameters of the model must be determined from experiment, either by fitting fatigue data or by devising special tests to measure the parameters individually. It is possible that some of the response functions of the model and the attendant parameters thereof might be determined by means of atomistic or mixed continuum/atomistic analysis of crack tips under cyclic loading. Work to this effect is presently underway and will be reported in the near future. 


\section{ACKNOWLEDGMENTS}

We are grateful for support provided by the Army Research Office through Caltech's MURI on Engineering Microstructural Complexity in Ferroelectric Devices.

\section{REFERENCES}

1. J. Nuffer, D. Lupascu, and J. Rödel, "Damage evolution in ferroelectric PZT induced by bipolar electric cycling," Acta Materialia 48, pp. 3783-3894, 2000.

2. D. Damjanovic, "Ferroelectric, dielectric and piezoelectric properties of ferroelectric thin films and ceramics," Reports on Progress in Physics 61, pp. 1267-1324, 1998.

3. Q. Jiang and L. Cross, "Effects of porosity on electric fatigue behaviour in PLZT and PZT ferroelectric ceramics," Journal of Material Science 28, pp. 4536-4543, 1993.

4. Q. Jiang, W. Cao, and L. Cross, "Electric fatigue in lead zirconate titanate ceramics," Journal of the American Ceramic Society 77, pp. 211-215, 1994.

5. J. Nuffer, D. Lupascu, and J. Rödel, "Microcrack clouds in fatigued electrostrictive 9.5/65/35 PLZT," Journal of the European Ceramic Society 21, pp. 1421-1423, 2001.

6. J. Nuffer, D. Lupascu, A. Glazounov, H.-J. Kleebe, and J. Rödel, "Microstructural modifications of ferroelectric lead zirconate titanate ceramics due to bipolar electric fatigue," Journal of the European Ceramic Society 22, pp. 2133-2142, 2002.

7. E. Burcsu, Investigation of Large Strain Actuation in Barium Titanate. PhD thesis, California Institute of Technology, 2001.

8. S.-J. Kim and Q. Jiang, "Microcracking and electric fatigue of polycrystalline ceramics," Smart Materials and Structures 5, pp. 321-326, 1996.

9. H. Cao and A. Evans, "Electric-field-induced fatigue crack growth in piezoelectrics," Journal of the American Ceramic Society 77, pp. 1783-1786, 1994.

10. C. Lynch, W. Yang, L. Collier, Z. Suo, and R. McMeeking, "Electric field induced cracking in ferroelectric ceramics," Ferroelectrics 166, pp. 11-30, 1995.

11. C. Lynch, L. Chen, Z. Suo, R. McMeeking, and W. Yang, "Crack growth in ferroelectric ceramics driven by cyclic polarization switching," Journal of Intelligent Material Systems and Structures 6, pp. 191-198, 1995.

12. T. Zhu, F. Fang, and W. Yang, "Fatigue crack growth in ferroelectric ceramics below the coercive field," Journal of Material Science Letters 18, pp. 1025-1027, 1999.

13. D. Fang, K. Ai, and J. Liu, "Electromechanical deformation and fracture of piezoelectric/ferroelectric materials," Acta Mechanica Sinica 17, pp. 193-213, 2001.

14. S. Park and S. Sun, "Effect of electric field of fracture of piezoelectric ceramics," International Journal of Fracture 70, pp. 203-216, 1995.

15. W. Yang and T. Zhu, "Switch-toughening of ferroelectrics subjected to electric fields," Journal of the Mechanics and Physics of Solids 46, pp. 291-311, 1998.

16. T. Zhu and W. Yang, "Toughness variation of ferroelectrics by polarization switch under non-uniform electric field," Acta Materialia 45, pp. 4695-4702, 1997.

17. T. Zhu and W. Yang, "Fatigue crack growth in ferroelectrics driven by cyclic electric loading," Journal of the Mechanics and Physics of Solids 47, pp. 81-97, 1999.

18. W. Yang, F. Fang, and M. Tao, "Critical role of domain switching on the fracture toughness of poled ferroelectrics," International Journal of Solids and Structures 38, pp. 2203-2211, 2001.

19. Z. Suo, C.-M. Kuo, D. Barnett, and J. Willis, "Fracture mechanics for piezoelectric ceramics," Journal of the Mechanics and Physics of Solids 40, pp. 739-765, 1992.

20. S. Li, "On saturation-strip model of a permeable crack in a piezoelectric ceramic," Acta Mechanica 165, pp. 47-71, 2003.

21. T.-Y. Zhang, M. Zhao, and P. Tong, "Fracture of piezoelectric ceramics," Advances in Appled Mechanics 38, pp. 147-289, 2001.

22. R. McMeeking, "Towards a fracture mechanics for brittle piezoelectric and dielectric materials," International Journal of Fracture 108, pp. 25-41, 2001. 
23. S. Hwang, C. Lynch, and R. McMeeking, "Ferroelectric/ferroelastic interactions and polarization switching model," Acta Metallurgica Et Materialia 43, pp. 2073-2084, 1995.

24. H. Gao, T.-Y. Zhang, and P. Tong, "Local and global energy release rates for an electrically yielded crack in a piezoelectric ceramic," Journal of the Mechanics and Physics of Solids 45, pp. 491-510, 1997.

25. C. Fulton and H. Gao, "Effect of local polarization switching on piezoelectric fracture," Journal of the Mechanics and Physics of Solids 49, pp. 927-952, 2001.

26. C. Fulton and H. Gao, "Microstructural modeling of ferroelectric fracture," Acta Materialia 49, pp. 20392054, 2001.

27. O. Nguyen, E. A. Repetto, M. Ortiz, and R. Radovitzky, "A cohesive model of fatigue crack growth," International Journal of Fracture 110(4), pp. 351-369, 2001.

28. S. Serebrinsky and M. Ortiz, "A cohesive model of fatigue crack nucleation in metals." (in preparation), 2004.

29. I. Arias, S. Serebrinsky, and M. Ortiz, "A cohesive model of fatigue crack nucleation in ferroelectrics." (in preparation), 2004.

30. M. Ortiz and A. Pandolfi, "Finite deformation irreversible cohesive elements for three-dimensional crackpropagation analysis," International Journal for Numerical Methods in Engineering 444, pp. 1267-1282, 1999.

31. X. Gong and Z. Suo, "Reliability of ceramic multilayer actuators: a nonlinear finite element simulation," Journal of the Mechanics and Physics of Solids 44, pp. 751-769, 1996.

32. C. Landis, "A new finite-element formulation for electromechanical boundary value problems," International Journal For Numerical Methods In Engineering 55, pp. 613-628, 2002.

33. Y. Shu and K. Bhattacharya, "Domain patterns and macroscopic behavior of ferroelectric materials," Philosophical Magazine B 81(12), pp. 2021-2054, 2001.

34. J. H. Rose, J. R. Smith, F. Guinea, and J. Ferrante, "Universal features of the equation of state of metals," Physical Review B 29(6), pp. 2963-2969, 1984.

35. J. Rice, "Dislocation nucleation from a crack tip: an analysis based on the Peierls concept," Journal of the Mechanics and Physics of Solids 40(2), pp. 239-271, 1992.

36. S. Takahashi, K. Okajima, and A. Hasegawa, "Fatigue test of piezoelectric ceramics under high hydrostatic pressure," Japanese Journal of Applied Physics 29, pp. 47-49, 1990.

37. Military standardization handbook - Metallic materials and elements for aerospace vehicle structures, MILHDBK-5H, U. S. Department of Defense, Washinton, D.C., 1998. 